\title{
APROXIMACIÓN AL ORIGEN Y EVOLUCIÓN HISTÓRICA DEL PENSAMIENTO ADMINISTRATIVO
}

Cpa. Edith Vanessa Bonín Campos Docente titular tiempo completo Instituto Superior Tecnológico de Formación edith.bonin@formación.edu.ec
Lic. Evelyn de la Llana Pérez Instituto Superior Tecnológico de Formación evelyn.delallana@formación.edu.ec

Palabras claves: administración, organización, empresas.

Recibido: 04 de octubre de 2016

Keywords: administration, organization, companies.

Aceptado: 11 de noviembre de 2016

\section{RESUMEN}

Las principales escuelas del pensamiento administrativo han contribuido a una mejor y mejor comprensión de las organizaciones por parte de los gerentes. Por la importancia que tienen en el desempeño organizacional contemporáneo, en el presente artículo se analizan las mismas. Cada una de ellas ofrece una perspectiva distinta para definir los problemas, las oportunidades y desarrollar distintas maneras de hacerles frente, por lo que se considera pertinente realizar este breve recorrido teniendo en cuenta el importante cúmulo de variables que revela para el análisis de los diversos problemas por los que atraviesan las empresas en el mundo actual.

\section{ABSTRACT}

The main schools of administrative thought have contributed to a better understanding of organizations by mangers. Because of the importance these schools have in the current organizational performance the present article will focus on them. Each of these schools gives a different perspective to define the problems, the opportunities and develop different ways of dealing with them. It can be considered pertinent to carry out this brief study taking into account the important accumulation of variables that reveals for the analysis of the various problems faced by companies in today's world. 


\section{INTRODUCCIÓN}

La administración es tan antigua como la misma humanidad, desde que existió un jefe y un subordinado se hizo palpable la necesidad de administrar. El pensamiento administrativo es muy antiguo, ya que nace con el hombre mismo, porque en cualquier tiempo de la historia ha habido la necesidad de coordinar, tomar decisiones y ejecutar. Sin embargo la historia de las organizaciones y su administración tiene su origen en una época más reciente. Al analizar la historia de la humanidad, se pueden encontrar los aportes que han plasmado los antiguos pueblos, como los griegos, romanos y egipcios. Conforme la evolución de la humanidad fue avanzando las personas empezaron a escribir sobre cómo lograr que las organizaciones fueran eficaces y eficientes, mucho antes de que se conociera el término de administración como lo conocemos en la actualidad. Fue así como la ciencia de la administración se erige como una herramienta indispensable para el correcto manejo de los diferentes recursos; materiales y humanos, dentro de cualquier labor que se realice en los diversos ámbitos de acción: industria, comerci.

\section{DESARROLLO}

\section{Escuela Clásica}

En el despuntar del siglo XX, dos ingenieros desarrollaron los primeros trabajos respecto a la administración. Frederick Winslow Taylor desarrolló la llamada escuela de administración científica, preocupada por aumentar la eficiencia de la industria a través, inicialmente, de la racionalización del trabajo operario. El otro Henri Fayol desarrolló la llamada teoría clásica preocupada por aumentar la eficiencia de su empresa a través de su organización y de la aplicación de principios generales de la administración con bases científicas. Las ideas y planteamientos de Fayol y Taylor constituyeron las bases del llamado enfoque clásico tradicional de la administración, cuyos postulados dominaron aproximadamente las cuatro primeras décadas de este siglo y el panorama administrativo de las organizaciones.

Frederick Winslow Taylor ingeniero industrial y economista estadounidense fue el promotor de la organización científica del trabajo y es considerado el padre de la Administración Científica. El mismo planteo que la administración científica involucra una completa evolución mental de parte de los trabajadores de cualquier establecimiento o industria específica, una revolución mental completa de los hombres en cuanto a la responsabilidad hacia su trabajo, hacia los demás hombres y hacia sus patrones. E involucra una igualmente completa revolución mental por parte de aquellos en el lado de la administración - el capataz, el superintendente, el dueño del negocio, la junta directiva - una completa revolución mental en cuanto a sus responsabilidades hacia sus compañeros de trabajo en la administración, hacia sus trabajadores y hacia todos sus problemas diarios. Y sin esta completa revolución mental en ambos lados la administración científica no existe. Y esta es la esencia de la administración científica, esta gran revolución mental.

Henri Fayol fue uno de los principales contribuyentes al enfoque clásico de la administración. Su aporte principal fue el de escribir sobre problemas no estudiados por Taylor, ya que mientras Taylor concentra sus estudios en el taller o la fábrica, Fayol lo hace a nivel de la dirección, creando lo que algunos llaman escuela de "jefes". Hizo grandes contribuciones a los diferentes niveles administrativos. Escribió Administration industrielle et générale, el cuál describe su filosofía y sus propuestas. Fayol plantea las funciones de la administración como: técnicas, comerciales, financieras, de seguridad, contables y administrativas. 
Dentro de las funciones distingue el proceso correcto de administración: Planear, organizar, dirigir, coordinar y controlar. Fayol afirma que la capacidad básica de las personas situadas en los niveles inferiores es la capacidad profesional característica de la empresa, mientras que la capacidad esencial de la alta dirección es la administrativa. Es decir, conforme se asciende en la escala jerárquica de la organización deben aumentar las funciones administrativas, mientras que si se desciende predominan las funciones técnicas.

\section{Escuela de las relaciones Humanas}

La Escuela de las Relaciones Humanas fue un movimiento de reacción y oposición a la Escuela Clásica de la organización. La misma mejoró la perspectiva clásica que considera a la producción como un asunto casi exclusivamente de ingeniería y se interesó en la gente como parte del proceso. La esencia de este movimiento era el concepto de que la clave para que las organizaciones tuvieran mayor productividad estaba en aumentar la satisfacción de los empleados. La escuela de las relaciones humanas pone énfasis en los elementos emocionales inconscientes del comportamiento humano.

El psicólogo de la escuela de administración de empresas de Harvard Elton Mayo fue el padre de las relaciones humanas. Inicio una nueva era en el desarrollo de la administración. Aplicó las ciencias del comportamiento a los conceptos de administración de grupos humanos. "Los problemas humanos en una civilización industrial” (1933), es su principal publicación. Buscó el incremento de la productividad por medio del análisis y el mejoramiento de las condiciones psicológicas y sociales del individuo. Demostró además la importancia que tiene en el rendimiento del obrero su estado de ánimo, la estructura informal de su grupo, el tipo de autoridad ejercida por su jefe y la mayor o menor participación en la determinación de su propio trabajo. Mediante estos estudios se incorpora a la administración la Psicología y la Sociología y se introduce la dinámica de grupo y la motivación individual como aspectos importantes.

Abrraham $\mathrm{H}$. Maslow fue un psicólogo humanista que buscó incrementar la productividad a través de la satisfacción personal del individuo (autorrealización). Su pirámide de las necesidades humanas permite comprender que existe una jerarquía de necesidades y cada vez que se satisface una, la siguiente adquiere un carácter dominante. Las necesidades que plantea el mismo son:

1) fisiológicas (comer, dormir)

2) seguridad (protección contra peligro y amenazas)

3) afiliación o sociales (ser aceptado, asociación)

4) estimación de sí mismo (confianza, autonomía, competencia)

5) autorrealización (consideración, nivel social)

Su publicación principal es "Motivation and personality" (1954)

Douglas Mc Gregor formuló dos series de teorías sobre la naturaleza humana. El mismo plantea que no existe una teoría satisfactoria de la administración, diciendo que el administrador supone el comportamiento de la gente y modifica la posición del gerente autocrático y unilateral supeditando las necesidades de la organización a las necesidades de los individuos. Se trata de aprovechar lo mejor de cada hombre; usar sus puntos fuertes y no sus puntos 
débiles. El mismo plantea que existen dos condiciones extremas del individuo: Teoría $X$ y Teoría Y. La Teoría X.- utilizada por la teoría clásica de la organización del trabajo: * No le gusta trabajar y hace todo por evitarlo. * trabaja únicamente por dinero y amenazas de castigo. * es irresponsable y carece de iniciativa; prefiere ser dirigido. La Teoría Y.- tiene iniciativa y es responsable. * ayuda para alcanzar los objetivos que considera valiosos. * es capaz de auto-controlarse y auto-dirigirse si acepta los objetivos de su trabajo. * tiene mayores posibilidades intelectuales que las que usa. Mc Gregor pensaba que los supuestos de la teoría "y" captaba mejor la verdadera naturaleza de los trabajadores y deberían guiar el ejercicio de la administración.

Frederick Herzberg desarrolló en 1972, en la unidad de negocios de la universidad de Utah, su teoría motivación- higiene. Con la posible excepción de los estudios Hawtorn, ninguna corriente de investigación aislada ha tenido mayor fuerza de impacto sobre las recomendaciones de la administración científica que la obra de Herzberg. Buscó desde los años cincuenta la respuesta a la pregunta: ¿Qué quieren los trabajadores de su empleo? Plantea que los factores de higiene:

1) políticas y administración de la empresa

2) clase de supervisión que la gente recibe

3) condiciones laborales y relaciones interpersonales

4) nivel de vida y seguridad

Aunque no conducen a mayores niveles de motivación, sin los mismos no hay satisfacción. Por su parte los factores de seguridad abarcan lo que la gente realmente hace en el trabajo:

1) logros

2) reconocimiento por logros

3) interés en las tareas

4) crecimiento, avance y responsabilidad por tareas mayores

Plantea que los dos aspectos deben atenderse simultáneamente para que la gente sienta un mínimo de insatisfacción y para que puedan crecer y avanzar en su trabajo

Robert R. Blake y J.S Mouton han estado presente en el mundo del cambio y desarrollo organizacional durante los últimos y son los creadores de un modelo bidimensional de estilo gerencial que tiene el propósito de que el director conozca sus propias tendencias gerenciales para mejorarlas o complementarlas; el interés por la gente en el eje vertical y el interés por la producción en el eje horizontal. Escogieron escalas de 9 puntos para describir su modelo y para calificar el grado de interés del gerente por la producción y por las personas. Uno representa un interés mínimo y 9 indica un gran interés. Hay 81 combinaciones posibles pero en forma realista decidieron considerar las cuatro posiciones extremas de las esquinas de la cuadrícula, y el estilo intermedio del centro de la misma.

La oposición de la teoría de las relaciones humanas- con su profundo énfasis en las personas- a la teoría clásica- centrado en las tareas y la estructura de la organización- derivó 
hacia una segunda etapa: la teoría del comportamiento que representó un nuevo intento para sintetizar la teoría de la organización formal con la escuela de las relaciones humanas. Con la llegada de este enfoque el énfasis de la estructura se desplazó hacia los procesos y la dinámica de las organizaciones, es decir, hacia el comportamiento de las personas en las organizaciones (desarrollo organizacional).

\section{Escuela Neoclásica}

El consultor en administración Peter F. Drucker expone en sus ideas el reflejo de todos los métodos de dirección que han significado el éxito de las empresas norteamericanas de los últimos 35 años. "The practice of management" (1954) y "Management for results (1964)", son sus publicaciones principales. Para Drucker la empresa es una institución concebida para crear cambios y ello significa satisfacer a las personas de afuera y obtener resultados afuera, satisfacer a los miembros de la organización no es la tarea principal ni el desafío de las organizaciones. Otras personas que pueden considerarse dentro de este enfoque son Ernest Dale y Harold Koontz. La Teoría Neoclásica se caracteriza por hacer gran énfasis en los aspectos prácticos de la administración, la reafirmación relativa de los principios clásicos, énfasis en los objetivos y en los resultados; y eclecticismo que toma contenidos de casi todas las teorías administrativas.

\section{Escuela Estructuralista. (Teoría de la Burocracia)}

A partir de la década de 1940 las críticas a la Escuela Clásica- por su mecanicismo- como a la Escuela de las Relaciones Humanas - por su romanticismo- dieron paso a una nueva teoría de la organización inspirada en los escritos de del economista y sociólogo alemán, ya entonces fallecido, Max Weber. Max Weber (1864-1920). Así como a Taylor le interesaba la administración en el taller- lo que actualmente se llama trabajo del supervisor- y a Fayol le interesaba centrarse en las funciones de la administración general, Weber formuló una teoría de las estructuras de la autoridad y describió las actividades de las organizaciones basadas en relaciones de autoridad. Describió una organización ideal que llamó burocracia. Trató de combinar la estructura formal con aspectos del comportamiento humano y la conexión de la organización con todo el sistema social.

Además cabe mencionar a Cyril N. Parkinson profesor de ciencias políticas que se hizo famoso por sus críticas humorísticas a los defectos de funcionamiento de las grandes organizaciones burocráticas. Por su parte Laurence J. Peter intentó demostrar que la acción administrativa revela la búsqueda de justificaciones para el desempeño ineficiente. Su libro "Principio de Peter" (1965) habla de la necesidad de conocer nuestros propios límites y niveles de incompetencia; pero como Parkinson, no encuentra relación directa entre el número de empleados y el volumen de trabajo realizado. Arthur Bloch en su libro "Ley de Murphy y otras razones por que las cosas salen mal”, indica que sí algo puede fallar, fallará y platea los siguientes corolarios:

\section{1. nada es tan fácil como parece serlo}

\section{2. todo tiene necesidad de más tiempo del que usted piensa}

3. si existe alguna posibilidad de que varias cosas puedan salir mal, saldrá mal aquello que cause el mayor daño

4. si se dejan al azar las cosas, tenderán a ir de mal en peor. 


\section{5. cada solución genera nuevos problemas}

6. es imposible hacer algo a prueba de tontos, porque éstos son muy ingeniosos.

Además aparecen leyes como la Ley de Gresham (enemiga de la planeación) Los asuntos sin importancia se resuelven rápidamente, (el dinero malo desplaza al bueno). La Ley de Pareto (80/20) La Ley de Weiler que plantea que nada es imposible para el hombre que no debe desempeñar ese trabajo. Y la Ley de Katz diciendo que los hombres y las naciones actuarán razonablemente cuando se agoten las demás posibilidades.

\section{Escuela Sistémica}

Es una consecuencia de la teoría general de sistemas desarrollada en la década de 1950 por el biólogo alemán Ludwing Von Bertalanffy. Esta teoría interdisciplinaria trasciende los problemas exclusivos de cada ciencia y proporciona principios- sean físicos, matemáticos, biológicos, psicológicos, etc.- y modelos generales para todas las ciencias involucradas, de modo que los descubrimientos efectuados en cada ciencia puedan utilizarlos las demás. De este modo, las diversas ramas del conocimiento- hasta entonces divorciadas unas de otras por su gran especialización- consideraron que sus objetos de estudios eran sistemas, incluso la administración. Algunos representantes de esta escuela son: Norbert Wiener y Herbert Simon.

Un sistema es el conjunto de piezas interdependientes, relacionadas entre sí y ordenadas de modo que producen un todo unificado. Hay dos tipos de sistemas: los sistemas cerrados que no están sujetos a la influencia de su entorno ni interactúan con él y los sistemas abiertos que reconocen la interacción dinámica entre el sistema y su entorno.

El comportamiento de las organizaciones es probabilista y no determinista, por formar parte de un sistema mayor. Las organizaciones no operan en forma aislada. Su supervivencia depende de la interacción correcta con el entorno, el cual abarca la situación económica, los mercados globales, las actividades políticas, los avances tecnológicos, las costumbres sociales y el trato con los grupos de interés de la organización. Esta escuela incluye al enfoque cuantitativo (investigación de operaciones) y requiere todavía de mejor sistematización y elaboración para su aplicación práctica.

\section{Escuela Situacional}

Con el advenimiento de la teoría situacional se desplaza la observación desde adentro hacia fuera de la organización. La teoría situacional planteó nuevos aspectos respecto a los conceptos de eficiencia y eficacia. Casi todas las teorías administrativas de enfoque prescriptivo y normativo, siguiendo el modelo de sistema cerrado se preocuparon únicamente por la eficiencia. La eficiencia no se preocupa por los fines sino sólo por los medios. Conseguir los objetivos fijados es competencia de la eficacia. La eficacia organizacional es la habilidad de la empresa para explorar su entorno, ya sea para obtener recursos o para llevarle sus productos. Los principales representantes de esta cuela son:

Kurt Lewin quien realizó investigaciones en la Universidad de Lowa para determinar las conductas que hacían exitoso a un líder y encontró 3 estilos: • autocrático • democrático • permisivo. Alfred D. Chandler por su parteen 1962 realizó una de las más serias investigaciones históricas sobre los cambios estructurales de las grandes organizaciones (DuPont, General Motors, Standard Oil y Sears) y los relacionó con la estrategia de negocios. 
El cambio del entorno es el factor principal para la elección de la estructura adecuada. Paul R. Lawrence y Jay W. Lorsch fueron los primeros teóricos de la contingencia. En 1972 hicieron una investigación sobre la relación causa - efecto entre lo bien que la estructura interna de una organización se adapta a las exigencias ambientales y cuán bien alcanza la organización sus metas y objetivos: Esta hipótesis originó la teoría situacional. Preocupados por las características que deben tener las empresas para enfrentar las diferentes condiciones externas, tecnológicas y de mercado; compararon 10 empresas en 3 sectores industriales (plásticos, alimentos empacados y contenedores). Concluyeron que los conceptos primordiales de su teoría son dos estados opuestos y antagónicos : la diferenciación- división en subsistemas o departamentos- y la integración - proceso opuesto para unificar los esfuerzos y la coordinación de los departamentos o subsistemas generados por presiones provenientes del entorno-. Cuanto mayor sea la complejidad ambiental, más complejo deberá ser el diseño interno. La solución no es que una organización deba estar altamente diferenciada o altamente integrada, sino en que las dos tengan ambos atributos.

\section{La excelencia empresarial.}

W. Edwards Deming en su libro "Out of the Crisis" (salida de la crisis), publicado en 1986, expone su teoría sobre la administración para alcanzar el mejoramiento de la calidad, la productividad y la posición competitiva. En sus catorce puntos el Dr. W. Edwards Deming hizo operativa su teoría de la administración de la calidad. La teoría de Deming de la Administración incluye el uso de herramientas estadísticas y técnicas del comportamiento.

El Dr. Joseph M. Juran responde la pregunta de muchos administradores, “¿para qué estoy aquí?". Él explica que los administradores tienen dos funciones básicas: a.- Romper los procesos existentes para llegar a nuevos niveles de rendimiento, y b.- Mantener los procesos mejorando en sus nuevos niveles de rendimiento. El enfoque situacional es eminentemente ecléctico e integrador, asimila los conceptos de las diversas teorías administrativas para ampliar los horizontes y mostrar que nada es absoluto. También cabe mencionar en este apartado a Akio Morita legendario empresario Japonés que hizo de la empresa electrónica Sony un gigante. Se centró en la mercadotecnia y su afán de ofrecer los productos más avanzados. Su publicación principal es "Made in Japan" (1986). Kaoru Ishikawa por su parte le da gran importancia al concepto de calidad total, que abarque a toda la institución, para lo cual se deben crear comités Interfuncionales y grupos de proyectos (círculos de calidad).

Wiliam Ouchi en su libro "Teoría Z" (1980), identifica mediante el estudio de grandes empresas Japonesas las características comunes: a) Gran identificación de la empresa con sus empleados. b) Tratamiento cuidadoso de la empresa a los empleados. c) Lealtad de los colaboradores hacia la empresa. d) Sistema de empleo de por vida. e) Baja rotación de personal. f) Enorme motivación por buenos resultados de productividad. g) Participación personal intensiva en todos los procesos administrativos.

Thomas J. Peters y Robert $\mathrm{H}$. Waterman de acuerdo a la investigación que realizaron en empresas Norteamericanas obtuvieron un común denominador de las empresas excelentes:
a) Ser el mejor
b) Saber hacer el trabajo bien
c) Recordar siempre la importancia del ser humano 
d) Lograr calidad en el producto y su servicio

Su publicación principal es "In search of excellence" (1982)

\section{CONCLUSIONES}

La administración no permanece sola como campo reconocido de conocimiento. Es una disciplina integral que se fundamenta y relaciona con diversas ciencias y técnicas tales como las ciencias sociales y humanas. La administración aprovecha las leyes y análisis de la Sociología para la coordinación de personas y cosas. Para el manejo de las "personas" se auxilia de la psicología, la ciencia que explica cómo opera la motivación en los actos humanos y consecuentemente la forma de predecirlos. Presta valiosa ayuda proporcionando el conocimiento de los factores de la personalidad del hombre. En la psicología se estudia la conducta humana en general y en la administración se aplican las técnicas psicológicas para obtener de los hombres eficacia y la máxima eficiencia dentro de una empresa. En otro sentido toma del Derecho ese conjunto de ordenamientos legales y jurídicos que rigen a la sociedad y regulan la conducta social de los individuos; pues sólo sobre la base de una justicia establecida por el derecho puede quedar asentada firmemente una estructura en concordancia con las actividades que regulan: civil, mercantil, laboral, fiscal y constitucional. De la Economía tomó la ley de obtener el máximo de resultados con el mínimo de esfuerzos (concepto de productividad)

La condición actual de la administración incluye ideas introducidas hace decenas de años. Los conceptos de una época no remplazan completamente a los de otra anterior, por el contrario, extienden y modifican ideas anteriores. Cada una de las teorías administrativas presentadas tiene un enfoque diferente que refleja los fenómenos históricos, sociales, culturales y económicos de su época. No se puede decir que una es más acertada que otra pues cada una de ellas presenta la solución o soluciones encontradas para determinadas circunstancias, teniendo en cuenta las variables identificadas y los temas más importantes dentro de la administración. 


\section{REFERENCIAS BIBLIOGRÁFICAS}

Alvarez, F. (2000). Principios de administración; $2^{\text {a }}$ edición; Ediciones Eudecor; Argentina.

Chivenato, I. (1997). Introducción a la Teoría General de la Administración; Cuarta Edición. Bogotá, Mc Graw Gill.

Koontz, H y Heinz, W. (1998). Administración, una perspectiva global; 11va Edición. Mc Graw Hill.

Martínez, M. (2004). Psicología Organizacional I. Selección de Lecturas. Parte I. La Habana: Editorial Félix Varela.

Martínez, M. (2004). Psicología Organizacional II. Selección de Lecturas. Parte I. La Habana: Editorial Félix Varela.

Maslow, H. (2005). El management según Maslow: una visión humanista para la empresa de hoy (orig.: Maslow on Management). Barcelona: Editorial Paidós Ibérica. ISBN 84-4931698-7.

Maslow, H. (1991). Motivación y personalidad. Madrid: Ediciones Díaz de Santos. ISBN 84-87189-84-9.

Medina, A. y Ávila, A. (2001). Desarrollo organizacional. Una visión desde la psicología organizacional. La Habana: Universidad de la Habana.

Peiró, J. (2005). Psicología de la Organización. Tomo I y II. Ed. Félix Varela. La Habana. 


\section{NORMAS PARA LAS PUBLICACIÓN DE ARTÍCULOS}

\section{I.- TIPO DE ARTÍCULOS}

1. Los artículos pueden ser:

- Informes de investigación

- Informes de desarrollo tecnológico

-Ensayo

- Resúmenes de tesis de grado

- Revisiones bibliográficas

- Propuestas de modelos e innovaciones educativas

\section{ESTRUCTURA DEL ARTÍCULO}

2-. Existe un límite de 8 páginas como mínimo y 18 como máximo para cada artículo

3-. El título estará en Times New Roman 12, en negrita, centrado, con mayúscula y procurando que no exceda las 10 palabras.

4-. Para efecto de identificación de la revista con el autor(es) del artículo, debajo del Título, centrado y sin negrita este detallará afiliación institucional(especificando sitio de trabajo), así como su dirección electrónica.

5-. El/los autor(es) deberá incluir en un párrafo, que no puede exceder las (80) palabras, un resumen de su currículo donde conste su formación profesional, trabajo actual, actividad investigativa y/o aportes académicos más destacados. En caso de ser 2 autores, a cada uno se le otorga un máximo de 50 palabras. Debe haber más autores, el resumen de cada curriculum séra reducido en función del espacio destinado en la revista a criterio del Comité Editorial.

6-. Todos los documentos deben contener un resumen escrito en español y en inglés, con un máximo de 200 palabras. El resumen debe mencionar el objetivo general, la metodología empleada cuando corresponda y señalar los principales resultados y/o conclusiones.

7-. Se deben indicar de tres a cinco descriptores o palabras clave más significativas del documento

8-. Para el desarrollo del cuerpo, se recomienda incluir alguno o todos los siguientes elementos:

- Introducción.

- Materiales y métodos (o Metodología).

- Resultados.

- Discusión (o Conclusiones).

9-.Todo trabajo deberá incorporar las referencias bibliográficas al final del documento según

el formato utilizado por la American Psychological Association (APA). 


\section{III.- FORMATO DEL ARTÍCULO}

10-. El documento debe estar escrito en Times New Roman, tamaño 12 , márgenes de 2,5 $\mathrm{cm}$ en todos los lados, justificado totalmente con interlineado simple.

11-. Las ilustraciones (figuras, tablas, gráficos) deberán enumerarse secuencialmente incluyendo en una descripción explicativa para cada una.

12-. Los títulos de figuras, tablas o gráficos deben ser de un tamaño inferior al texto normal

13-. Los títulos o apartados que componen el escrito deben aparecer en negrita

\section{DE LA EVALUACIÓN DEL ARTÍCULO}

14-. Los artículos se dividen en tres categorías:

- Ensayos académicos: revisiones bibliográficas, ensayos o propuestas de modelos e innovaciones educativas.

- Artículo técnicos: informes de desarrollo tecnológico, o resúmenes de tesis de grado.

- Artículo científico: informes de investigación.

Para cada caso, se asignará una valoración de acuerdo a los parámetros expuestos en la página web del departamento de investigación: www.formacion .edu.ec/investigacion. 